Rev. Elev. Méd. vét. Pays trop., 1977, 30 (3) : 281-292

\title{
Caractéristiques biologiques des hématies des bovins de la zone tropicale de l'Afrique de l'Ouest
}

\author{
par P. CUQ (*), A. J. AKAKPO $\left(^{* *}\right)$ et D. FRIOT $\left(^{* * *}\right)$
}

\begin{abstract}
RÉSUMÉ
Les auteurs étudient les caractéristiques biologiques des érythrocytes des bovins (zébus et taurins) du Sénégal et du Togo. La morphologie, la numération, l'hématocrite, le taux d'hémoglobine, la résistance globulaire et les index de Wintrobe sont tout à tour examinés ainsi que leurs variations en fonction de l'espèce, de la race, de l'état sexuel, de l'àge et de la saison. Les résultats sont comparés à ceux d'autres populations bovines de l'Afrique et des régions tempérées.
\end{abstract}

Les caractéristiques biologiques des érythrocytes des bovinés des régions tempérées ont fait l'objet de nombreuses publications. Celles qui se rapportent aux bovins de la zone tropicale sèche de l'Afrique de l'Ouest sont par contre très rares. A l'exception des données partielles que l'on trouve dans les travaux de FRIOT et CALVET (8) au Sénégal et de GAULIER (10) à Madagascar, les quelques autres documents dont on peut disposer proviennent des pays anglophones : VOHRADSKY (20) au Ghana, ODUYE et OKUNAIYA (14) au Nigéria, HASSAN et HOELLER (12) au Soudan et SMITH (18) en Ouganda. Les renseignements que l'on peut en tirer sont toujours fragmentaires et souvent même contradictoires. C'est la raison pour laquelle le laboratoire d'Anatomie, Histologie et Embryologie de l'Ecole Inter-Etats des Sciences et Médecine Vétérinaires de Dakar a entrepris, depuis 1973, cette étude consacrée aux zébus et aux taurins du Sénégal et du Togo.

(*) Professeur, Ecole Inter-Etats des Sciences et Médecine Vétérinaires B.P. 5077, Dakar (Rép. du Sénégal).

(**) Assistant, Ecole Inter-Etats des Sciences et Médecine Vétérinaires B. P. 5077, Dakar (Rép. du Sénégal).

(***) Laboratoire National d'Elevage et de Recherches Vétérinaires. I. S. R. A., B. P. 2057, Dakar-Hann, (Rép. du Sénégal).

\section{MATERIEL ET METHODES}

1) Le travail a porté sur 413 zébus et 226 taurins.

Sur les 413 zébus, 304 vivaient au Sénégal et parmi eux 271 étaient de race Gobra (79 mâles entiers, 124 mâles castrés et 68 femelles) et 33 de race Maure $(3$ mâles entiers, 17 mâles castrés et 13 femelles). Les 109 zébus du Togo appartenaient au groupe Peulh du Sahel (particulièrement aux races White Fulani, Sokoto Gudali et Mbororo et à leurs croisements). Ils comprenaient 44 mâles entiers, 58 mâles castrés et 7 femelles.

Sur les 226 taurins, 122 provenaient du Sénégal et étaient de race Ndama (21 mâles entiers, 94 mâles castrés et 7 femelles) et 104, de races lagunaires ou Muturu, provenaient du Togo (12 mâles entiers, 69 mâles castrés et 23 femelles).

2) Ces animaux ont été divisés en 5 classes d'âge évalué sur les critères de chronologie dentaire de GILIBERT (11) pour le zébu et de DUMAS et LHOSTE (7) pour les taurins.

La classe $A$ comprend les animaux de moins de 2 ans $1 / 2$ ( 2 ans à 2 ans $1 / 2$ pour les zébus et 1 an $1 / 2$ à 2 ans $1 / 2$ pour les taurins). 
La classe B correspond aux animaux de 2 ans $1 / 2$ à 4 ans $1 / 2$.

La classe $C$ à ceux de 4 ans $1 / 2$ à 6 ans $1 / 2$ pour les zébus et à ceux de 4 ans $1 / 2$ à 7 ans pour les taurins.

La classe D comprend les zébus de 6 ans $1 / 2$ à 10 ans et les taurins de 7 à 10 ans.

La classe $E$ à ceux qui ont plus de 10 ans.

3) Les prélèvements du Togo ont été effectués pendant la période des grandes vacances universitaires (15 juillet-15 octobre), c'est-àdire pendant une période où l'eau et l'herbe sont particulièrement abondantes.

Ceux du Sénégal ont été réalisés pendant la période d'activité scolaire (15 octobre-15 juillet) qui correspond à 4 saisons et 4 périodes d'alimentation différentes :

- octobre et novembre se situent à la fin de la saison des pluies, période chaude où l'herbe et l'eau peuvent être consommées à satiété ;

- décembre, janvier et février, à la saison fraîche ou "post-hivernage " pendant lequel l'alimentation est toujours abondante mais de plus en plus sèche ;

- en mars et avril, période de printemps, certains arbres et arbustes se couvrent de pousses et de jeunes feuilles qui sont appétées par les animaux et améliorent leur ration ;

- en mai, juin et juillet, saison sèche, l'alimentation est de plus en plus ligneuse et de plus en plus rare.

4) Le sang a été recueilli par ponction veineuse, au laboratoire, ou après section de la veine jugulaire externe, à l'abattoir.

Ce mode de prélèvement présente l'avantage de permettre en une seule fois la récolte d'une quantité de sang suffisante pour plusieurs examens. Il évite la dilution du sang capillaire par la lymphe du pavillon de l'oreille ou par les sécrétions des glandes naso-labiales du mufle et évite aussi sa pollution par des débris tégumentaires qui compliquent les examens et risquent de fausser les résultats.

Deux anti-coagulants ont été successivement utilisés : le citrate de soude à 3,8 p. 100 à raison de $0,1 \mathrm{ml}$ par $\mathrm{ml}$ de sang et l'E. D. T. A. (sel di-sodique pur de l'acide ethylène-diaminetétracétique) à raison de $0,1 \mathrm{mg}$, par $\mathrm{ml}$ de sang.
5) Les frottis ont été colorés par la méthode panoptique avec le colorant de May GrunwaldGiemsa (MG-G) selon la technique décrite par RULLIER et PARODI (16) et par GABE (9), mais en utilisant le tampon phosphate à $0,1 \mathrm{M}$

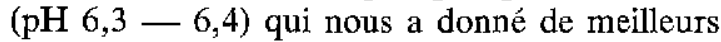
résultats que le tampon à $\mathrm{pH} 6,5-6,8$ préconisé par GABE, ou l'eau distillée à $\mathrm{pH} 7$ préconisée par RULLIER et PARODI.

Pour détecter certaines formes d'hématies, des colorations particulières ont été employées: solution alcoolique de bleu de Crésyl brillant à 0,5 p. 100 , sulfate de bleu de Nil à 1 p. 100 dans l'alcool absolu, solution de bleu de toluidine à 1 p. 100 dans l'alcool méthylique, solution de bleu de méthylène à 0,5 p. 100 dans l'alcool méthylique.

6) Les numérations ont été effectuées à l'hématimètre de Neubauer, à Lomé. A Dakar, nous avons utilisé successivement l'hématimètre de Malassez puis un numérateur automatique de marque Picoscale fabriqué par Hellige qui a été mis gracieusement à notre disposition par le Professeur LINHARD, Directeur du Centre de Transfusion Sanguine du Sénégal que nous tenons à remercier ici. La formule de correction des valeurs lues au compteur du numérateur Picoscale a été calculée à partir de la table fournie par le fabricant pour les numérations globulaires de l'homme jusqu'à la valeur 6,99 du compteur qui est insuffisante pour les animaux. Elle est : $\mathrm{Y}_{\mathrm{GR}}=0,000070$ $\mathrm{x}^{2}+1,040 \mathrm{x}+0,7$ avec $\mathrm{x}$ et $\mathrm{y}$ exprimés en multiples de $10^{4}$ globules rouges lus au compteur ( $\mathrm{x}$ ) et en valeur corrigée (y).

7) Le taux d'hémoglobine a été évalué par la méthode à la cyanmethémoglobine.

8) L'hématocrite a été évalué par la méthode du micro-hématocrite plus précise et plus fidèle que la méthode de Wintrobe pour le sang des bovins dont les globules sédimentent très mal en raison du faible pouvoir d'agglutination des hématies de cette espèce.

9) La résistance globulaire a été mesurée par l'action hémolysante de solutions salées de concentration croissante. Les concentrations d'hémolyse initiale (H. I.) et d'hémolyse totale (H. T.) ont été relevées.

10) Le traitement statistique des résultats a été réalisé par le service d'informatique de 
l'Institut Universitaire de Technologie de Dakar. Les documents qui s'y rapportent sont consignés dans la thèse de Doctorat-Vétérinaire de l'un d'entre nous (2).

\section{RESULTATS}

Les résultats concernent, la morphologie des globules rouges, leur numération, l'hématocrite, le taux d'hémoglobine, la résistance globulaire et les index érythrocytaires de Wintrobe.

\section{MORPHOLOGIE DES ERYTHROCYTES DES BOVINS TROPICAUX}

Il n'existe pas de différence morphologique importante entre les globules rouges des zébus et des taurins du Sénégal et du Togo que nous avons étudiés et ceux des bovidés des régions tempérées.

Leur FORME est comme pour la plupart des mammifères, celle d'un disque légèrement biconcave. Après coloration au MG-G leur aspect est parfaitement homogène et la pâleur centrale que l'on observe dans certaines espèces est toujours faible ou nulle.

Leur DIAMETRE est en moyenre 5,62 $\mu$, c'est-à-dire un peu plus grand que celui des taurins des régions tempérées $(5,5 \mu)$. Comme dans ces derniers, on observe souvent une subanisocytose qui se traduit par la présence dans le même sang d'érythrocytes dont les diamètres varient de $4,5 \mu$ à $6,75 \mu$ et, parfois seulement, une anisocytose vraie dans laquelle on trouve à côté des normocytes $(5,65 \%$ de diamètre), des microcytes $(3,5$ à $4,5 \mu$ de diamètre) et des mégalocytes $(9,0$ à 10,1 : de diamètre). Chez les zébus et les taurins que nous avons étudiés, la subanisocytose est plus fréquente pendant la saison sèche que pendant la saison des pluies et l'anisocytose vraie est de règle dans les anémies graves.

Comme ceux des bovins des régions tempérées, les érythrocytes des bovins de l'Afrique tropicale ont un très faible pouvoir d'agglutination et les regroupements en "rouleaux * sont inexistants.

La poïkylocytose est rare. Les sélenocytes et les échinocytes que nous avons observés correspondent le plus souvent à des artefacts.

Les anomalies de coloration sont aussi très rares. Nous n'avons pu observer que quelques hématies vacuolisées et quelques cellules cibles (Target-cells) dont le centre coloré est entouré d'un anneau clair lui-même circonscrit par le bord cellulaire coloré.

Las formes immatures sont normalement totalement absentes chez les taurins et les zébus que nous avons étudiés. Les réticulocytes sont en particulier toujours absents dans le sang de ces animaux, qui tous étaient âgés de plus d'un an et demi. Chez les bovins des régions tempérées, environ 0,1 p. 100 des adultes en possèdent.

Les formes immatures peuvent par contre être nombreuses lors des érythropoièses accélérées qui accompagnent les grandes anémies. Nous avons ainsi observé :

- des érythrocytes polychromatophiles, toujours grands $(10 \mu)$ et dont le cytoplasme est, par zone, bleuté ou orangé après coloration au MG-G;

- des réticulocytes de 6 à 7,5 $\mu$, dans le cytoplasme (plus ou moins basophile) desquels subsistent quelques organites ;

- des érythroblastes basophiles de $12 \mu$ de diamètre dont les noyaux intensément basophiles ont perdu leurs nucléoles et présentent une chromatine épaisse disposée en amas radiaires ;

- des érythroblastes polychromatophiles de 8 à $12 \mu$ et à cytoplasme violacé ou gris brun sale;

— des érythroblastes acidophiles de $6,8 \mu$ avec un noyau réduit à une petite tache violette et un cytoplasme rouge orangé ;

— et des corps de Jolly, restes nucléaires violacés, sphériques et excentriques qui persistent dans des globules rouges d'apparence normale.

\section{NUMERATION ERYTHROCYTAIRE}

\section{A. Valeurs moyennes des numérations érythrocytaires}

Les difficultés matérielles qui ont été la conséquence d'un travail effectué parallèlement au laboratoire et à l'abattoir d'une part, au Togo et au Sénégal d'autre part, nous ont conduit à utiliser trois appareils différents pour les numérations : les hématimètres de Neubauer 
et de Malassez et le numérateur automatique Picoscale.

Afin de regrouper et de comparer de façon valable les résultats fournis par chacun d'eux, nous avons évalué le degré de précision des mesures fournies et recherché par analyse statistique s'il était possible de regrouper les résultats.

Le contrôle de précision a été réalisé en pratiquant trente numérations érythrocytaires avec le même sang et par le même opérateur, avec chacun des appareils. Le calcul des intervalles de confiance, des variations, celui des valeurs extrêmes par rapport à la moyenne et la recherche des distributions de fréquences par rapport à la moyenne ont permis de conclure que les résultats obtenus par l'hémaLimètre de Malassez (intervalle de confiance $\pm 0,09$, pourcentages des valeurs extrêmes 5,51 p. 100 et 5,80 p. 100 et distribution symétrique) étaient plus précis que ceux de l'hématimètre de Neubauer (intervalle de confiance $\pm 0,15$, pourcentage des valeurs extrêmes, 5,14 p. 100 et 12,32 p. 100 et distribution légèrement déportée à droite) et que ceux du numérateur Picoscale (intervalle de confiance $\pm 0,09$, pourcentages des valeurs extrêmes 4,13 p. 100 et 8,62 p. 100 et distribution fortement déviée à gauche).

Les possibilités de regroupement des résultats ont été testées en effectuant cinq séries de cinq numérations du même sang avec chaque appareil. Après calcul des moyennes ( $\overline{\mathrm{x}})$, des intervalles de confiance (I. C.) et de la significativité à 5 p. 100 des différences entre résultats par analyse de la variance (test de F), l'analyse statistique a montré qu'il n'existe pas de différence significative entre les résultats fournis par les hématimètres mais que trois fois sur cinq ceux du numérateur automatique diffèrent significativement des deux autres.

Nous traiterons donc à part les résultats fournis par l'appareil Picoscale.

Dans le tableau $\mathrm{n}^{0} \mathrm{I}$, les résultats sont exprimés en $1.10^{6}$ érythrocytes. Il en sera de même pour la discussion.

\section{B. Discussion}

\section{1) Influence de l'espèce et de la race}

L'analyse de la variance par comparaison deux à deux montre qu'il n'y a pas de différence significative entre les taurins et les zébus du Sénégal (pour les deux séries à comptage automatique respectivement $8,12 \pm 0,24$ pour les Ndama, $8,07 \pm 0,32$ pour les Gobra et $8,06 \pm 0,65$ pour les Maures) mais qu'il existe une différence hautement significative entre les taurins et les zébus du Togo (pour les deux séries à numération par hématimètre : taurins lagunaires 7,56 $\pm 0,34$ et zébus du groupe Peulh du Sahel, 6,62 $\pm 0,31$ ).

TABLEAU N $\mathrm{N}^{\circ} \mathrm{I}$-Numérations êrythrocytaires.

\begin{tabular}{|c|c|c|c|c|}
\hline & $\begin{array}{c}\text { Moyenne gênérale } \\
\text { N et X } \pm \\
\text { I.C. }\end{array}$ & $\begin{array}{c}\text { Mâles castrës } \\
\text { N et X } \pm \\
\text { I.C. }\end{array}$ & $\begin{array}{l}\text { Feme'lles } \\
N \text { et X } \pm \\
\text { I.C. }\end{array}$ & $\begin{array}{c}\text { Mäles entiers } \\
\text { Net X } \pm \\
\text { I.C. }\end{array}$ \\
\hline \multicolumn{5}{|l|}{ Hêma ti mè tre } \\
\hline Taurins du Toga & $\begin{array}{c}7,56 \pm 0,34 \\
(104)\end{array}$ & $7,83_{(69)} \pm 0,36$ & $6,85 \pm \frac{(20,91}{(23)}$ & $\begin{array}{c}7,39 \pm 1,25 \\
(12)\end{array}$ \\
\hline Zébus du Togo & $\begin{array}{c}6,62 \pm 0,31 \\
(109)\end{array}$ & $\begin{array}{c}6,69 \pm 0,42 \\
(58)\end{array}$ & $\begin{array}{c}6,89 \pm 1,43 \\
\text { (7) }\end{array}$ & $\begin{array}{c}6,49 \pm 0,52 \\
(44)\end{array}$ \\
\hline Zébus Gobra & $\begin{array}{c}7,87 \pm 0,32 \\
(79)\end{array}$ & $\begin{array}{c}8,04 \pm 0,43 \\
(50)\end{array}$ & $\begin{array}{c}7,24 \pm 0,68 \\
(18)\end{array}$ & $\begin{array}{c}8,41 \pm 0,69 \\
(11)\end{array}$ \\
\hline Zébus Maure & $\begin{array}{c}8,06 \pm 0,76 \\
\text { (12) }\end{array}$ & & & \\
\hline \multicolumn{5}{|l|}{ Picoscale } \\
\hline Taurins Ndama & $\begin{array}{c}8,12 \pm 0,24 \\
(106)\end{array}$ & $\begin{array}{c}8,13 \pm 0,25 \\
(83)\end{array}$ & $\begin{array}{c}7,61 \pm 0,73 \\
(7)\end{array}$ & $\begin{array}{c}8,32 \pm 0,99 \\
\text { (16) }\end{array}$ \\
\hline Zébus Gobra & $\begin{array}{c}8,07 \pm 0,32 \\
(192)\end{array}$ & $\begin{array}{c}8,07 \pm 0,32 \\
(74)\end{array}$ & $\begin{array}{c}8,23 \pm 0,40 \\
(50)\end{array}$ & $\begin{array}{c}7,95 \pm 0,32 \\
(68)\end{array}$ \\
\hline Zébus Maure & $\begin{array}{c}8,06 \pm 0,71 \\
(21)\end{array}$ & & & \\
\hline
\end{tabular}


L'examen des résultats fournis par les zébus du Togo montre que le lot qu'il constitue est très hétérogène $\left(2,7\right.$ à $\left.10,1.10^{6}\right)$ et que les classes les plus nombreuses sont à peu près équivalentes et comprises entre 5,6 et 9,5.10 . Cette particularité s'explique par le fait que le groupe des zébus du Togo est constitué par le rassemblement de plusieurs races et de leurs croisements. Ainsi une partie d'entre eux présente une numération érythrocytaire voisine de $8.10^{6}$, comme les zébus du Sénégal ou les zébus du Soudan (HASSAN et collab. (12), ADAM et collab. (1)) tandis que l'autre, inférieure à $7.10^{6}$, est plus proche des valeurs données par SMITH (18) pour les femelles zébus d'Ouganda $\left(6,21.10^{(i)}\right.$ et par GAULIER (10) pour le zébu Malgache $\left(4,84 \cdot 10^{6}\right)$. Les taurins du Sénégal et du Togo présentent donc des numérations érythrocytaires voisines et proches de celles des zébus du Sénégal et d'une partie seulement des zébus du Togo. Le reste des zébus Peulh du Sahel présente des numérations plus faibles.

\section{2) Influence du sexe}

Il n'existe aucune différence significative entre les valeurs de numération des différents groupes sexuels. Nous avons remarqué cependant, comme l'ont fait HASSAN et collab. (12) pour les zébus du Soudan, que chez les taurins de races lagunaires les mâles castrés ont une numération un peu plus élevée $\left(7,83.10^{\circ}\right)$ que les femelles $\left(6,85 \cdot 10^{6}\right)$. Le sexe a peu d'influence sur la numération érythrocytaire. Dans les deux espèces cependant elle tend à être un peu plus élevée chez les mâles castrés.

\section{3) Influence de l'âge}

L'analyse statistique met en évidence quelques différences significatives entre classes d'âge mais elles correspondent toujours à des classes peu nombreuses dont les sujets présentent des déviations importantes, par excès et défaut, par rapport à la moyenne. Ces différences mathématiques ne correspondent donc pas à un caractère biologique constant. L'âge a donc peu d'influence sur la numération érythrocytaire des bovins de l'Afrique tropicale de l'Ouest.

Cependant comme SCHALM (17) et WYERS (21) l'ont fait pour les bovins des régions tempérées, nous pouvons noter que chez les taurins du Togo et du Sénégal les moyennes baissent au fur et à mesure que l'animal vieillit. La numération érythrocytaire des taurins a donc tendance à diminuer avec l'âge dont l'influence est faible dans l'ensemble.

\section{4) Influence de la saison}

L'influence de la saison a été étudiée sur les zébus Gobra du Sénégal. Bien que l'effectif correspondant soit important (192 animaux), l'analyse statistique par comparaison de la variance deux à deux ne révèle aucune différence significative. On peut cependant noter une baisse régulière des moyennes de la fin de la saison des pluies $\left(8,45.10^{6}\right)$ au post hivernage $\left(8,41.10^{6}\right)$, au printemps $\left(8,01.10^{5}\right)$ et à la saison sèche $\left(7,51 \cdot 10^{6}\right)$. La numération érythrocytaire tend donc à diminuer au cours de la saison sèche.

\section{HEMATOCRITE}

L'hématocrite a été étudié sur les bovidés du Sénégal seulement.

A. Valeurs moyennes de l'hématrocrite des bovidés du Sénégal (voir tabl. $n^{\circ}$ II)

TABLEAU N ${ }^{\circ}$ II-Microhématocrite des bovidés du Sênêgal.

\begin{tabular}{|c|c|c|c|c|}
\hline Mi c rohëma tocri te & $\begin{array}{c}\text { Moyennes générales } \\
\mathrm{N} \text { et } \mathrm{X} \pm \\
\text { I.C. }\end{array}$ & $\begin{array}{c}\text { Mâles castrés } \\
\text { X et } X \pm \\
\text { I.C. }\end{array}$ & $\begin{array}{l}\text { Femelles } \\
\text { N et } \mathrm{X} \pm \\
\text { I.C. }\end{array}$ & $\begin{array}{c}\text { Mâles entiers } \\
\text { N et X } \pm \\
\text { I.C. }\end{array}$ \\
\hline Taurins (Ndama) & $\begin{array}{c}36,5 \pm 1,2 \\
(106)\end{array}$ & $\begin{array}{c}36,0 \pm 1,5 \\
(83)\end{array}$ & $\begin{array}{c}35,0 \pm 4,1 \\
(7)\end{array}$ & $\begin{array}{c}34,0 \pm 3,5 \\
(16)\end{array}$ \\
\hline Zébus Gobra & $\begin{array}{c}37,8 \pm 1,3 \\
(192)\end{array}$ & $\frac{38,0 \pm 1,2}{(74)}$ & $\begin{array}{c}37,0 \pm 1,3 \\
(50)\end{array}$ & $\begin{array}{c}37,0 \pm 1,5 \\
(68)\end{array}$ \\
\hline Zébus Maure & $\begin{array}{c}36,8 \pm 2,6 \\
(2 i)\end{array}$ & & & \\
\hline
\end{tabular}




\section{B. Discussion}

\section{1) Influence de l'espèce}

Bien que l'analyse de la variance ne révèle pas de différence significative entre les taurins Ndama et les zébus Gobra, on peut noter que l'hématocrite du Ndama est sensiblement plus bas $(36,5)$ que celui du Gobra $(37,8)$. Cette remarque est confirmée par FRIOT et CALVET (8) qui, travaillant sur des effectifs beaucoup plus nombreux, mettent en évidence une différence significative entre les deux espèces. Les chiffres donnés par ces auteurs sont légèrement plus faibles que les nôtres (34,7 pour le Ndama et 37,5 pour le Gobra). Cette différence peut être rapportée aux techniques employées, aux conditions de récoltes et au fait que l'hématocrite du sang prélevé au moment de la saignée est un peu plus élevé que celui qui est effectué sur l'animal qui reste en vie (3). Les mêmes remarques sont valables pour les valeurs données pour la West African Shorthorn (35,1 ; VOHRADSKY (19)), les Ndama du Ghana $(34,2$; VOHRADSKY (19)) et du Nigeria $(30,1$; ODUYE et collab. (14)) et le zébu du Soudan $(36,7$; ADAM et collab. (1)).

En Afrique tropicale lhématocrite du zébu est donc un peu plus élevé que celui des taurins.

\section{2) Influence du sexe}

Bien qu'il n'existe pas de différence significative entre les groupes sexuels des bovins du Sénégal que nous avons étudiés, nous pouvons remarquer que l'hématocrite est sensiblement plus élevé chez les Ndamas et les zébus Gobra mâles castrés $(36,0$ et 38,0$)$ que chez les femelles $(35,0$ et 37,0$)$ et les mâles entiers $(34,0$ et 37,0$)$. Cette remarque est corroborée par FRIOT et CALVET (8) qui ont montré sur des effectifs plus importants que l'hématocrite des mâles castrés est significativement plus élevé que celui des femelles et des mâles entiers.

\section{3) Influence de l'âge}

L'analyse de la variance met en évidence une différence significative entre les zébus Gobra mâles entiers âgés de 6 ans $1 / 2$ à 10 ans et les autres classes d'âge. Ce groupe, peu nombreux (6 individus), comprend 5 sujets à hématocrite élevé $(44,33,52$ et 49$)$ et dont les prélèvements ont été effectués en novembre, saison pendant laquelle l'hématocrite est sensiblement plus fort. Il semble donc qu'avec CHARPENTIER et BONHOMME (5) nous devions conclure que l'âge est sans influence mais, FRIOT et CALVET (8) travaillant sur des effectifs plus importants que les nôtres ont montré que l'hématocrite atteint une valeur maximale entre 2 et 4 ans et diminue ensuite peu à peu.

L'hématocrite atteint donc une valeur maximale chez les jeunes adultes. Il diminue ensuite peu à peu avec l'âge.

\section{4) Influence de la saison}

Appliquée à l'hématocrite du zébu Gobra, l'analyse de la variance ne rèvèle pas de différence significative sur les lots que nous avons examinés. Nous pouvons remarquer cependant que les valeurs décroissent de la fin de l'hivernage $(38,0)$ au post-hivernage $(37,0)$ et au printemps $(37,0)$ et à la saison des pluies $(36,0)$. FRIOT et CALVET (8), sur des effectifs beaucoup plus importants, ont montré qu'il existe une différence significative entre l'hématocrite du post-hivernage $(39,9)$ et de l'hivernage $(39,9)$ et celui de la saison sèche $(38,4)$. Il est vraisemblable, comme le pensent COURCEL (6) et PAYNE et collab. (15), que ces variations soient à rapporter à l'abreuvement et à l'alimentation.

Comme la numération érythrocytaire, l'hématocrite est donc plus élevé en saison des pluies qu'en saison sèche.

\section{TAUX D'HEMOGLOBINE}

Le taux d'hémoglobine (exprimé en $g$ p. $100 \mathrm{ml}$ ) a été évalué chez les taurins et les zébus du Togo et du Sénégal.

\section{A. Valeurs moyennes des taux d'hémoglo- bine (voir tabl. $\mathrm{n}^{\circ}$ III)}

\section{B. Discussion}

\section{1) Influence de l'espèce}

Il n'existe aucune différence significative entre les valeurs respectives des deux espèces que nous avons étudiées. Nous pouvons remarquer cependant que les zébus du Sénégal $(11,8$ 
TABLEAU NIII-Taux d'hërnog lobine

\begin{tabular}{|c|c|c|c|c|}
\hline $\begin{array}{l}\text { Hémoglobine G } \\
\text { p.100 }\end{array}$ & $\begin{array}{c}\text { Moyennes génërales } \\
X \pm I . C .\end{array}$ & $\begin{array}{c}\text { Mâles castrés } \\
X \pm \text { I.C. }\end{array}$ & $\begin{array}{l}\text { Feme1les } \\
\mathrm{X} \pm \mathrm{I} . \mathrm{C} .\end{array}$ & $\begin{array}{c}\text { Mâles entiers } \\
\mathrm{X} \pm \text { I.c. }\end{array}$ \\
\hline Taurins du Togo & $\begin{array}{c}12,0 \pm 0,4 \\
(104)\end{array}$ & $\begin{array}{c}12,4 \pm 0,5 \\
(69)\end{array}$ & $\begin{array}{c}11,4 \pm 1,1 \\
(23)\end{array}$ & $\begin{array}{c}10,9 \pm 1,5 \\
(12)\end{array}$ \\
\hline Taurins du Sénēgal & $\begin{array}{c}11,1 \pm 0,4 \\
(106)\end{array}$ & $\begin{array}{c}11,2 \pm 0,4 \\
\langle 83\rangle\end{array}$ & $\begin{array}{c}10,9 \pm 1,3 \\
\text { (7) }\end{array}$ & $\frac{10,5 \pm 1,2}{(16)}$ \\
\hline Zébus du Togo & $\begin{array}{c}10,9 \pm 0,4 \\
(109)\end{array}$ & $\begin{array}{c}11,2 \pm 0,6 \\
(58)\end{array}$ & $\begin{array}{c}11,0 \pm 2,5 \\
\text { (7) }\end{array}$ & $\begin{array}{c}10,6 \pm 0,7 \\
(44)\end{array}$ \\
\hline $\begin{array}{l}\text { ZébusGobra du } \\
\text { Sénégal }\end{array}$ & $\begin{array}{c}11,8 \pm 0,5 \\
(192)\end{array}$ & $\begin{array}{c}12,2 \pm 0,5 \\
(74)\end{array}$ & $\begin{array}{c}11,7 \pm 0,5 \\
(50)\end{array}$ & $11,3 \pm 0,5$ \\
\hline $\begin{array}{l}\text { Zêbus Maure du } \\
\text { Sénégal }\end{array}$ & $11,6 \pm 0,9$ & & & \\
\hline
\end{tabular}

Zébus Maure, 20 colonne, ajouter : (21).

pour le Gobra et 11,6 pour le Maure) ont des taux d'hémoglobine plus élevés que les taurins Ndama $(11,1)$ alors qu'au Togo la différence est inverse (10,9 pour les zébus et 12,0 pour les taurins).

Travaillant sur des effectifs plus importants que les nôtres, FRIOT et CALVET (8) ont montré qu'il existe une différence significative entre les zébus Gobra $(11,6)$ et les taurins Ndama $(10,5)$.

Les zébus Gobra et Maures ont un taux d'hémoglobine plus élevé que les taurins Ndama. Les résultats particuliers du Togo s'expliquent par l'influence de la race.

\section{2) Influence de la race}

a) Il n'existe pas de différence significative entre les taux d'hémoglobine des taurins lagunaires du Togo $(12,0)$ et ceux du Ndama du Sénégal $(11,1)$. Ces valeurs sont en outre voisines de celles qui ont été déterminées chez les Ndama du Ghana $(11,87$; VOHRADSKY (19)) et chez la vache Ndama du Nigeria $(12,5$; ODUYE et collab. (14)). Elles sont aussi proches de celles de la West African Shorthorn (12,52 ; VOHRADSKY (19)) mais plus élevées que les taux moyens des bovins des régions tempérées $(11,0$; SCHALM (17)).

L'espèce, la race et la localisation n'ont donc pas d'effet sur le taux d'hémoglobine des taurins d'Afrique tropicale de l'Ouest. VOHRADSKY (20) estime qu'il est déterminé par le climat tropical et cite à l'appui de sa thèse le cas de vaches frisonnes anglaises dont le taux d'hémoglobine a augmenté en arrivant au Ghana. b) Bien qu'il n'existe pas de différence significative lorsque l'analyse de la variance par comparaison deux à deux est effectuée sur les lots de zébus que nous avons étudiés, nous pouvons remarquer que les taux d'hémoglobine des zébus du Sénégal $(11,8$ pour les Gobra et 11,6 pour les Maures) sont plus élevés que ceux des zébus du Togo $(10,9)$ et que le tableau des fréquences de ces derniers présente deux maximums, l'un à 9,9 et l'autre à 11,9 . Cette particularité s'explique par le fait qu'au Togo une partie des zébus correspond à des croisements entre les races Sokoto Gudali et White Fulani dont les taux d'hémoglobine ont été respectivement évalués à 11,68 (VOHRADSKY (19)) et 9,04 (ODUYE et collab. (14)). Aucun chiffre n'a été publié pour la race Mbororo. Les taux d'hémoglobine des zébus de races Maure et Gobra sont voisins et à peu près équivalents à ceux des zébus du Soudan $(11,7$; ADAM et collab. (1)) et à ceux de la race Sokoto Gudali. Ils sont aussi voisins mais légèrement inférieurs à ceux des zébus de Madagascar (12,33 GAULIER (10)) mais par contre nettement supérieurs à ceux des zébus d'Ouganda $(8,98$; SMITH (18)) plus proches des White Fulani du Togo.

Il existe donc d'importantes différences dans les taux d'hémoglobine des diverses populations zébus d'Afrique tropicale de l'Ouest.

\section{3) Influence du sexe}

Sur les lots que nous avons étudiés, l'analyse de la variance par comparaison orthogonale montre qu'il existe une différence significative entre les taux d'hémoglobine des zébus Gobra mâles castrés $(12,2)$ et les deux autres 
catégories sexuelles (11,7 pour les femelles et 11,3 pour les mâles entiers), ainsi qu'entre ceux des taurins du Togo mâles castrés $(12,4)$ et les autres catégories sexuelles (11,4 pour les femelles et 10,9 pour les mâles entiers).

Bien qu'aucune différence significative ne soit mise en évidence, la même remarque peut être faite pour les zébus du Togo (11,2 pour les mâles castrés, 11,0 et 10,6 pour les femelles et les mâles entiers) et pour les taurins Ndama du Sénégal (11,2 pour les mâles castrés, 10,9 et 10,5 pour les femelles et los mâles entiers).

Cette particularité est confirmée par les résultats obtenus par FRIOT et CALVET (8) sur le zébu Gobra. Elle infirme ceux de BYERS et collab. (4) et HOLLMAN et collab. (13) qui estiment que l'état sexuel n'a aucune influence sur le taux d'hémoglobine.

Pour l'ensemble des bovinés d'Afrique tropicale, le taux d'hémoglobine des mâles castrés est un peu plus élevé que celui des femelles et des mâles entiers.

\section{4) Influence de l'âge}

a) L'analyse de la variance par comparaison des classes deux à deux révèle une différence significative entre les classes A (1,5 à 2,5 ans), $\mathrm{D}$ (7 à 10 ans) et $\mathrm{E}$ (plus de 10 ans) des taurins du Togo mâles castrés et une autre différence significative entre les classes $\mathrm{D}(6,5$ à 10 ans) et $\mathrm{A}$ (1 à 2,5 ans) des zébus du Togo mâles entiers. Dans les deux cas, ces différences se rapportent à des classes très peu nombreuses et dont les sujets présentent de très grands écarts par excès et défaut par rapport à la moyenne. Ces différences mathématiques ne se traduisent done pas par une différence biologique caractéristique.

b) Chez les zébus Gobra il existe aussi une différence significative entre les mâles entiers de la classe D (4,5 à 6,5 ans) dont le taux d'hémoglobine est 13,5 et les autres classes d'âge dont les taux d'hémoglobine sont plus faibles. Sur les six animaux qui composent cette classe, 4 ont des taux d'hémoglobine élevés $(14,1 ; 15,4 ; 14,3$ et 15,4$)$ et correspondent à des prélèvements effectués à la fin de l'hivernage, saison pendant laquelle le taux d'hémoglobine comme l'hématocrite sont plus élevés.

c) Cependant, FRIOT et CALVET (8) sur des effectifs plus nombreux ont montré qu'au Sénégal, le taux d'hémoglobine comme l'hématocrite des taurins et des zébus croit jusqu'à 2 à 4 ans puis diminue ensuite.

Le taux d'hémoglobine atteint donc son maximum au début de lâge adulte. Il décrô̂t ensuite régulièrement.

\section{5) Influence de la saison}

L'influence de la saison a été étudiée sur les zébus Gobra du Sénégal. Le test statistique de comparaison deux à deux ne révèle pas de différence significative. Cependant, nous avons noté que le taux d'hémoglobine est plus élevé en saison des pluies $(12,1)$ et au printemps qu'en saison sèche $(11,3)$ et qu'au posthivernage $(11,4)$, ce que confirment FRIOT et CALVET (8) au Sénégal et SMITH (18) en Ouganda.

Le taux d'hémoglobine est donc plus élevé en saison des pluies et au printemps qu'en saison sèche et en post-hivernage.

\section{RESISTANCE GLOBULAIRE}

Le test de résistance globulaire a été réalisé sur des bovins du Sénégal et du Togo. Les valeurs correspondant aux résistances globulaires maximales (hémolyse totale) et aux résistances globulaires minimales (hémolyse initiale) sont exprimées en grammes pour mille de concentration en $\mathrm{Na} \mathrm{Cl}$.

\section{A. Valeurs moyennes de la résistance glo- bulaire (H. I. et H. T.) (voir tabl. $\mathrm{n}^{\circ}$ IV)}

\section{B. Discussion}

\section{1) Influence de l'espèce et de la race}

a) Il n'existe pas de différence significative des valeurs de résistances globulaires maximales et minimales entre les taurins du Togo et du Sénégal. Les taurins européens ont une valeur de résistance globulaire maximale $(4,8)$ nettement supérieure à celle des taurins d'Afrique, mais leur résistance globulaire minimale est plus faible $(6,2)$.

b) Il n'existe pas non plus de différence significative entre les valeurs de résistance globulaire des zébus du Sénégal et du Togo. Les chiffres que nous avons obtenus sont plus éle- 
TABLEAL $\mathrm{N}^{\circ} \mathrm{IV}$

\begin{tabular}{|c|c|c|c|c|}
\hline & & $\mathrm{N}$ & $\mathrm{H} T$ en $\underset{\mathrm{m} 1}{\mathrm{~g}} \mathrm{p} \cdot 100$ & H I $\underset{\text { ml }}{\text { H }}$ P.100 \\
\hline $\begin{array}{l}\text { Taurins } \\
\text { Lomé }\end{array}$ & $\begin{array}{l}\text { Moyennes gënërales } \\
\text { Mâles cas trếs } \\
\text { Femelles } \\
\text { Mâles entiers }\end{array}$ & $\begin{array}{r}104 \\
69 \\
23 \\
12\end{array}$ & $\begin{array}{l}3,93 \pm 0,07 \\
3,91 \pm 0,08 \\
3,94 \pm 0,20 \\
3,96 \pm 0,20\end{array}$ & $\begin{array}{l}7,21 \pm 0,06 \\
7,23 \pm 0,08 \\
7,21 \pm 0,15 \\
7,09 \pm 0,19\end{array}$ \\
\hline $\begin{array}{l}\text { Taurins } \\
\text { Dakar }\end{array}$ & $\begin{array}{l}\text { Moyennes générales } \\
\text { Mâles castrés } \\
\text { Femelles } \\
\text { Mâles entiers }\end{array}$ & $\begin{array}{r}106 \\
83 \\
7 \\
16\end{array}$ & $\begin{array}{l}3,73 \pm 0,06 \\
3,73 \pm 0,07 \\
3,71 \pm 0,27 \\
3,68 \pm 0,15\end{array}$ & $\begin{array}{l}6,87 \pm 0,10 \\
6,90 \pm 0,11 \\
7,14 \pm 0,32 \\
6,61 \pm 0,36\end{array}$ \\
\hline $\begin{array}{l}\text { Zêbus } \\
\text { Lomé }\end{array}$ & $\begin{array}{l}\text { Moyennes gênérales } \\
\text { Mâles cas três } \\
\text { Femelles } \\
\text { Mâles entiers }\end{array}$ & $\begin{array}{r}109 \\
58 \\
7 \\
44\end{array}$ & $\begin{array}{l}4,10 \pm 0,07 \\
4,13 \pm 0,09 \\
3,94 \pm 0,39 \\
4,08 \pm 0,11\end{array}$ & $\begin{array}{l}7,26 \pm 0,07 \\
7,33 \pm 0,11 \\
7,08 \pm 0,46 \\
7,20 \pm 0,09\end{array}$ \\
\hline $\begin{array}{l}\text { Zêbus } \\
\text { Gobra } \\
\text { Dakar }\end{array}$ & $\begin{array}{l}\text { Moyennes générales } \\
\text { Mâles castrës } \\
\text { Femelles } \\
\text { Mâles entiers }\end{array}$ & $\begin{array}{r}192 \\
74 \\
50 \\
68\end{array}$ & $\begin{array}{l}4,13 \pm 0,11 \\
4,14 \pm 0,13 \\
4,03 \pm 0,12 \\
4,21 \pm 0,09\end{array}$ & $\begin{array}{l}7,16 \pm 0,12 \\
7,21 \pm 0,10 \\
7,19 \pm 0,16 \\
7,07 \pm 0,13\end{array}$ \\
\hline
\end{tabular}

vés que ceux que rapporte GAULIER (10) pour les zébus Malgaches (3,64 et 6,67).

c) Bien que le test statistique de l'analyse de la variance ne révèle pas de différence significative entre les valeurs moyennes globales de résistance globulaire, celles des zébus $(4,11$ et 7,21 ) sont légèrement supérieures à celles des taurins $(3,88$ et 7,02$)$.

En Afrique tropicale, les zébus ont donc souvent des valeurs de résistance globulaire légèrement supérieures à celles des taurins.

\section{2) Influence du sexe et de l'âge}

Il n'y a pas d'influence du sexe ou de l'âge sur les valeurs de résistance globulaire.

\section{3) Influence de la saison}

L'influence de la saison a été testée sur les zébus Gobra. L'analyse de la variance ne révèle pas de différence significative. Cependant on peut noter que la résistance globulaire maximale augmente de façon régulière de la saison des pluies $(3,90)$ au post-hivernage $(3,99)$, au printemps $(4,24)$ et à la saison sèche $(4,42)$.

La résistance globulaire maximale tend donc à s'accrôitre en saison sèche.

\section{INDEX ERYTHROCYTAIRES DE WINTROBE}

Les index érythrocytaires de Wintrobe correspondent à des rapports numériques qui concernent le volume globulaire moyen, la teneur globulaire moyenne en hémoglobine et la concentration érythrocytaire moyenne en hémoglobine. Pour les animaux du Togo, sur lesquels l'hématocrite n'a pas pu être évalué, seule la teneur globulaire moyenne en hémoglobine a été calculée.

Les valeurs que nous présentons ont été calculées à partir de moyennes d'espèces et de races précédemment établies. Pour chaque catégorie, elles sont pratiquement identiques à celles qu'on obtient pour les divers sous groupes et peuvent donc être tenues pour valables pour l'ensemble de la population concernée.

\section{1) Volume globulaire moyen}

Le volume globulaire moyen exprime le rapport entre le volume globulaire total (hématocrite) ramené à $1 \mathrm{~mm}^{3}$ de sang et le nombre d'érythrocytes par $\mathrm{mm}^{3}$. 
TABLEAU N $\mathrm{N}^{\circ} \mathrm{V}$

\begin{tabular}{|l|c|c|c|}
\hline & $\begin{array}{c}\text { Hêmatocri te } \\
\text { rapporté } \\
\text { à } 1 \mathrm{man}^{3}\end{array}$ & $\begin{array}{c}\text { G.R. } / \mathrm{mm}^{3} \\
\text { en } 10^{6}\end{array}$ & VGM en $\mu^{3}$ \\
\hline $\begin{array}{l}\text { Taurins } \\
\text { Dakar }\end{array}$ & $36,5.10^{7}$ & $8,12.10^{6}$ & 44,9 \\
\hline $\begin{array}{l}\text { Zêbus } \\
\text { Dakar }\end{array}$ & $37,8.10^{7}$ & $8,07.10^{6}$ & 46,8 \\
\hline
\end{tabular}

Les taurins Ndama du Sénégal ont une valeur globulaire moyenne inférieure à celle des zébus Gobra mais les deux chiffres sont inférieurs à celui que rapporte SCHALM (17) pour les bovins européens : $52 \mu^{3}$.

\section{2) Teneur globulaire moyenne en hémoglobine}

La teneur globulaire moyenne en hémoglobine est le rapport entre la quantité d'hémoglobine contenue dans $1 \mathrm{~mm}^{3}$ de sang et le nombre d'érythrocytes dans le même volume.

\begin{tabular}{|c|c|c|c|}
\hline & $\mid \begin{array}{c}\text { IÉ mog lobine } \\
\times 10^{12}\end{array}$ & $\begin{array}{l}\text { G.R. } \\
\times 10^{11}\end{array}$ & $V . G .\left(\begin{array}{ll}\mu \mu & G\end{array}\right)$ \\
\hline $\begin{array}{l}\text { 'l'aurins } \\
\text { Lomê }\end{array}$ & 12,0 & 7,56 & 15,9 \\
\hline $\begin{array}{l}\text { Taurins } \\
\text { Dakar }\end{array}$ & 11,1 & 8,12 & 13,6 \\
\hline $\begin{array}{l}\text { Zêbus } \\
\text { Lomé }\end{array}$ & 10,9 & 6,62 & 16,5 \\
\hline $\begin{array}{l}\text { Zébus } \\
\text { Dakar }\end{array}$ & I1, 8 & 8,07 & 14,6 \\
\hline
\end{tabular}

Les bovins du Togo ont une valeur globulaire supérieure à celle des bovins du Sénégal.

\section{3) Concentration érythrocytaire moyenne en hémoglobine}

La concentration érythrocytaire moyenne en hémoglobine (C. E. M. H.) est la quantité d'hémoglobine contenue dans une unité de volume d'érythrocyte. Elle exprime le rapport entre la quantité d'hémoglobine contenue dans un volume donné de sang et le volume d'érythrocyte correspondant :

LABLEAU N ${ }^{\circ}$ VII

\begin{tabular}{|c|c|c|c|}
\hline & $\begin{array}{c}\text { Taux Hb.g } \\
\text { (en p. 100) }\end{array}$ & $\begin{array}{c}\text { Héma tocri te } \\
\text { (en p.100) }\end{array}$ & C.E.M.H. \\
\hline Ndama & 11,1 & 36,5 & 30,4 \\
\hline Gobra & 11,48 & 37,84 & 31,2 \\
\hline
\end{tabular}

Les taurins et les zébus du Sénégal ont des C. E. M. H. comparables et voisines de celles des bovins européens $(32,7)$.

\section{CONCLUSION}

Les érythrocytes des taurins et des zébus du Togo et du Sénégal ont une morphologie voisine de ceux des bovins des régions tempérées. Comme eux, ils présentent souvent une légère anisocytose et possèdent un très faible pouvoir d'agglutination qui rend les \& images en rouleaux $\gg$ très rares. Le sang des bovins d'Afrique est par contre toujours dépourvu de réticulocytes. Ces derniers et les formes immatures n'apparaissent que lors des érythropoièses accélérées qui accompagnent les grandes anémies.

Comparativement aux bovins des régions tempérées, les valeurs moyennes des constantes sanguines des taurins et zébus du Sénégal et du Togo s'établissent comme indiqué au tabl. $\mathrm{n}^{\circ}$ VIII.

L'influence de l'espèce et de la race est nette pour une partie des zébus togolais qui présente une numération érythrocytaire plus proche de celle des zébus d'Ouganda et de Madagascar que de celle des autres races de zébus et des taurins de l'Afrique tropicale de l'Ouest. De même, les taux d'hémoglobine des zébus se situent à deux niveaux différents : ceux du Gobra, du Maure, du zébu du Soudan, du Sokoto Gudali et du zébu de Madagascar qui sont nettement supérieurs à ceux du WhiteFulani et du zébu d'Ouganda. L'hématocrite et les valeurs de résistance globulaires des zébus sont un peu plus élevés que ceux des taurins.

L'influence du sexe se traduit par une nette tendance des mâles castrés à présenter une numération érythrocytaire, un hématocrite et un taux d'hémoglobine plus élevé que les femelles et les mâles entiers.

L'influence de l'âge est significative pour l'hématocrite et le taux d'hémoglobine qui augmentent de la naissance à l'âge adulte puis diminuent ensuite progressivement. De même, mais de façon plus discrète, la numération érythrocytaire tend à baisser avec l'âge chez les taurins.

L'influence de la saison se traduit par 
TABLEAL $\mathrm{X}^{\circ}$ VIII

\begin{tabular}{|c|c|c|c|c|c|}
\hline & $\begin{array}{l}\text { Zébus du } \\
\text { Sénégal }\end{array}$ & $\begin{array}{l}\text { Zëbus du } \\
\text { To\&c }\end{array}$ & $\begin{array}{l}\text { Taurins } \\
\text { du Sênêgal }\end{array}$ & $\begin{array}{l}\text { Taurins } \\
\text { du Togo }\end{array}$ & $\begin{array}{c}\text { Bovıns de } \\
\text { Regions tempérềes } \\
\text { (Schalm, 1971) }\end{array}$ \\
\hline $\begin{array}{l}\text { Nurération éry throcy taire } \\
\text { (hêmatimètre sauf pour } \\
\text { taurins du Sénéga1) }\end{array}$ & $\begin{array}{l}67,87.10^{6} \\
\text { M } 8,06.10^{6}\end{array}$ & $6,62 \cdot 10^{6}$ & $8,12 \cdot 10^{6}$ & $7,56 \cdot 10^{6}$ & $7.10^{6}$ \\
\hline $\begin{array}{l}\text { Microhématocrite } \\
(\mathrm{p}, 100)\end{array}$ & $\begin{array}{l}\text { M } 36, B \text { P } .100 \\
\text { Q } 37,6 \text { P. } 100\end{array}$ & & $36,5 \mathrm{p}, 1$ & & $35 \mathrm{p} \cdot 100$ \\
\hline $\begin{array}{l}\text { Taux d'hémog lobine en } \\
\text { g/ } 100 \mathrm{ml} \text {. }\end{array}$ & $\begin{array}{l}\text { M } 11,6 \mathrm{~g} \\
\text { G } 11,8 \mathrm{~g}\end{array}$ & $10,9 \mathrm{~g}$ & $11,1 \mathrm{~g}$ & $12.0 \mathrm{~g}$ & $11 \mathrm{~g}$ \\
\hline $\begin{array}{r}\text { Résis tance globulaire:HT } \\
\text { HI }\end{array}$ & $\begin{array}{l}4,13 \\
7,16\end{array}$ & $\begin{array}{l}4,10 \\
7,26\end{array}$ & $\begin{array}{l}3,73 \\
6,87\end{array}$ & $\begin{array}{l}3,93 \\
7,21\end{array}$ & $\begin{array}{l}4,8 \\
6,2\end{array}$ \\
\hline $\begin{array}{l}\text { Volume globularre } \\
\text { moyen }\end{array}$ & $46,8 \mu^{3}$ & & $44,9 \nu^{3}$ & & $52 \mu^{3}$ \\
\hline $\begin{array}{l}\text { Teneur g lobulaire } \\
\text { moyenne en hëmog looine }\end{array}$ & $14,6 \mu \nu \mathrm{g}$ & $16,5 \mu \mu g$ & $13,6 \mathrm{~g}$ & $15,9 \mu \nu \mathrm{g}$ & $14 \mu \mathrm{g}$ \\
\hline $\begin{array}{l}\text { Concentration érythrocy- } \\
\text { taire moyenne }\end{array}$ & 31,2 & & 30,4 & & 32,7 \\
\hline
\end{tabular}

$\mathrm{C}=$ Gobra ; $\mathrm{M}=$ Maure.

une diminution de la numération érythrocytaire et de l'hématocrite de la saison des pluies à la saison sèche. A l'inverse, la résistance globulaire maximale (H. T.) est plus élevée en sai- son sèche. Le taux d'hémoglobine passe par un maximum en hivernage et au printemps. Il est plus faible en saison sèche et en posthivernage.

\section{SUMMARY}

\section{Biological characteristics of cattle erythrocytes} in west African tropical area

The authors study the biological characteristics of Senegal and Togo cattle (Bos taurus and Bos indicus). Morphology, blood count, haematocrit, hemoglobin level, resistance of red blood corpuscles and Wintrobe's index are successively examined as also their vanation according to species, breed, sexual condition, age and season. The results are compared with those of other cattle from Africa and temperate areas.

\section{RESUMEN}

\section{Características biologicas de los eritrocitos de los bovinos de la zona tropical de Africa del Oeste}

Los autores estudian las características biologicas de los eritrocitos de los bovinos (cebues y taurinos) de Senegal y de Togo. Sucesivamente se examinaron la morfologia, la numeración, el hematocrito, la tasa de hemoglobina, la resistencia globular y los indices de Wintrobe así como sus variaciones en función de la especie, de la raza, del estado sexual, de la edad y de la estación. Se comparan los resultados con los de otras poblaciones bovinas de Africa y de las regiones templadas.

\section{BIBLIOGRAPHIE}

1. ADAM (S. E. I.), OBEID (H. M.) et TARTOUR (G.). Serum enzyme activities and haematology of normal and diseased ruminants in the Sudan. Acta Vet., Brno, $1974,43: 225-235$.

2. AKAKPO (A. J. B.). Contribution à l'étude de l'hématologie des bovins de l'Afrique de l'Ouest. Thèse Doct. vét. Dakar, 1976, $\mathrm{n}^{\circ} 14$.
3. BEAUDRY (J. C.). Variations de certains paramètres sanguins du cheval et du bœuf au moment de l'abattage. Thèse Doct. vét. Lyon, 1974, $\pi^{\circ} 68$.

4. BYERS (J. H.), JONES (I. R.) et HAAG (J. R.), Blood hemoglobin values of dairy cattle. J. Dairy Sci., 1952, $35: 661$.

5. CHARPENTIER (J.) et BONHOMME (D.). Fac- 
teurs de variation de l'hématocrite des bovins. I. Hématocrite des veaux à 8 jours. II. Hématocrite des femelles adultes. Annls Zootech., 1968, 17: 321326 et $327-335$.

6. COURCEL (B.). Constantes biochimiques sanguines de la vache laitière. Thèse Doct. vét., Lyon, 1972, $\mathrm{n}^{\circ} 18$.

7. DUMAS (R.), LHOSTE (P.). Les signes de l'âge chez le zébu. Etude des incisives de remplacement. Rev. Elev. Méd. vét. Pays trop., 1966, 13 : 357-363.

8. FRIOT (D.), CALVET (H.). Biochımie et élevage au Sénégal, Rev. Elev. Méd. vét. Pays trop., 1973, 26 : $75 a-96 a$.

9. GABE (M.). Techniques histologiques. Paris, Masson et Cie, 1968.

10. GAULIER (R.). Etude biochimique, biophysique et cytologique du sang de zébus malgaches (animaux d'abattoir). Rev. Elev. Méd. vét. Pays trop., 1970, 23 : 469-477.

11. GILIBERT (J.). Evolution des incisives chez les zébus malgaches. Rev. Elev. Méd. vét. Pays trop., 1974, $27: 115-123$.

12. HASSAN (Y. M.) et HCLLER (H.). Observations of the blood composition in cattle and sheep in Sudan. I. Cellular blood elements. Sudan J. yet. Sci. anim. Husb., 1966, 7 : 1-9.

13. HOLLMAN (H. H.), DEW (S. M.), BRACE-
WELL (C. D.). The blood picture in steers following oral dosing with Dictyocaulus viviparus and during a latent infection with virus pneumonia. Brit. vet. $J$., 1967, $123: 349-358$.

14. ODUYE (O. O.) et OKUNAIYE (O. A.). Etudes hématologiques chez les races bovines White Fulani et Ndama. Bull. epizoot. Dis. Afr., 1971, $19: 213-219$.

15. PAYNE (J. M.), DEW (S. M.), MANSTON (R.), FAULKS (M.). The use of a metabolic profile test in dairy herds. Vet. Rec., 1966, $87: 150-158$.

16. RULLIER (J.) et PARODI (A.). Laboratoire et diagnostic en médecine vétérinaire. Paris, Vigot Frères, 1968.

17. SCHALM (O. W.). Veterinary hematology. Philadelphia, Lee and Febiger, 1971.

18. SMITH (J. M.). The blood picture of normal zebu cows in Uganda. Brit. vet. J., 1959, 115: 89-96.

19. VOHRADSKY (F.). Variations diurnes de la formule sanguine de vaches Shorthorn-Ouest-Africain, Ndama, Sokoto-Gudali au Ghana. Acta Vet. Brno, $1971,40: 387-395$.

20. VOHRADSKY (F.). Observations sur quelques constantes sanguines chez le bétail Frison anglais importé au Ghana. Acta Vet. Brno, 1974, 43 : 221-224.

21. WYERS (M.). Normes hématologiques des animaux domestiques. Bull. Ass. fr. Vét. Microbiol. Immunol. Spéc. Mal. Infect., 1973 (12) : 51-67. 